\title{
Caspofungin may effective for initial treatment of pulmonary aspergillosis
}

\author{
Alper Sener, Senol Kobak, Timucin Alar, \\ Ugur Gonlugur, Asli Muratli
}

\begin{abstract}
Introduction: Invasive aspergillosis mostly involves lungs. Patients may present with fever, cough, hemoptysis and many other sign and symptoms. Underlying conditions that compromises pulmonary and immune responses to inhaled Aspergillus species serve as risk factors for pulmonary invasive aspergillosis. We are presenting a case of simple aspergilloma, successfully treated by caspofungin and surgery. Case Report: A 64year-old male patient has rheumatoid arthritis for ten years. Since three months he had interstitial pulmonary disease secondary to rheumatoid arthritis and hemoptysis. We performed microbiologic and pathologic investigations of the brochoalveolar lavage after computerized tomography for diagnosis of aspergillosis. Caspofungin was used for initial treatment of simple aspergilloma and also for follow-up treatment after apical wedge
\end{abstract}

Alper Sener ${ }^{1}$, Senol Kobak², Timucin Alar ${ }^{3}$, Ugur Gonlugur ${ }^{4}$, Asli Muratli 5

Affiliations: ${ }^{1}$ Onsekiz Mart University Faculty of Medicine, Infectious Disesae Department Terzioglu campus Canakkale/ Turkey; ${ }^{2}$ Onsekiz Mart University Faculty of Medicine, Rheumatology and Immunology Department Terzioglu campus Canakkale/Turkey; ${ }^{3}$ Onsekiz Mart University Faculty of Medicine, Thorasic Surgery Department Terzioglu campus Canakkale/Turkey; ${ }^{4}$ Onsekiz Mart University Faculty of Medicine, Pulmonary Disease Department Terzioglu campus Canakkale/Turkey; ${ }^{5}$ Onsekiz Mart University Faculty of Medicine, Pathology Department Terzioglu campus Canakkale/ Turkey.

Corresponding Author: Alper Sener, Onsekiz Mart University Faculty of Medicine, Infectious Disesae Department Terzioglu campus Canakkale/ Turkey; Email: dr.alpersener@gmail.com

Received: 12 June 2012

Accepted: 20 July 2012

Published: 01 March 2013 resection. The patient was discharged from the hospital at eighth week on itraconazol antifungal therapy. Conclusion: Three classes of drugs are available for the treatment of aspergillosis: polyens, azoles and echinocandins. Echinocandins have activity against Aspergillus spp. But have a limited role because they have not been studied for initial treatment of invasive aspergillosis.

Keywords: Antifungal treatment, Aspergillosis, Caspofungin

$* * * * * * * * *$

Sener A, Kobak S, Alar T, Gonlugur U, Muratli A. Caspofungin may effective for initial treatment of pulmonary aspergillosis. International Journal of Case Reports and Images 2013;4(3):161-164.

$$
* * * * * * * * *
$$

doi:10.5348/ijcri-2013-03-285-CR-6

\section{INTRODUCTION}

Aspergillus spp. may cause a variety of pulmonary diseases, depending on immune status and the presence of underlying lung disease [1]. Aspergillus species are ubiquitous in nature and inhalation of infectious conidia is a frequent event. Tissue invasion is uncommon and occurs most frequently in the setting of immunosuppression associated with therapy for hematologic malignancies, hematopoietic cell transplantation or solid organ transplantation [2]. Most invasive infections are caused by members of A. fumigatus species complex. Caspofungin is an echinocandin class antifungal drug. Echinocandins target fungal cell glucan synthesis by competitively inhibiting the beta-1,3-D-glucan synthase enzyme complex in susceptible fungi. This enzyme complex is 
composed of at least two components: catalytic subunits called Fks1p and Fks2p, and a GTP-binding protein, Rho1, which regulate the activity of glucan synthesis [3]. Echinocandins are widely used for the treatment of invasive candidiasis, especially in critically ill and neutropenic patients and less commonly in salvage regimens for invasive aspergillosis [4, 5]. Recent studies conclude that surgical resection should be considered for all patients with pulmonary aspergilloma who have acceptable pulmonary reserve. Echinocandin class of antifungal drugs have not been studies adequately as initial antifungal treatment of pulmonary aspergillosis. In this case we used caspofungin alone for initial treatment of pulmonary aspergilloma before and after surgery.

\section{CASE REPORT}

A 64-year-old male patient presented to us with hemoptysis for one week. He was a farmer by profession and had rheumatoid arthritis for ten years. He was taking prednisone $20 \mathrm{mg}$ per day for last three months for interstial pulmonary disease secondary to rheumatoid arthritis and azathioprine $100 \mathrm{mg}$ per day for anti-CCP positivity for last one month. He had fifteen days of cough and productive sputum and was given amoxicillin-clavulonate $2 \mathrm{gm}$ per day by his family practitioner. He was referred to our hospital with hemotysis of one week duration. The complete blood cell counts and blood biochemistry were within normal range except for high erythrocyte sedimentation rate (110 $\mathrm{mm} / 1 \mathrm{st} \mathrm{hr}$ ) and high $\mathrm{C}$ reactive protein (13.7 $\mathrm{mg} / \mathrm{dL}$ ). Chest radiograpgh showed a suspicious mass in the right apical lobe. Bronchoscopy were done for investigating tuberculosis by acid fast staining of brochoalveolary lavage (BAL). Computed tomography (CT) scan was performed because of suspicious mass on chest radiograph. There was a $5.8 \times 5.1 \times 8.6 \mathrm{~cm}$ right apical pleural based cavitary lesion with thick irregular wall. It was forming a small air cresent (Figure 1). Asid fast stain of BAL was negative. Routine staining of sputum and BAL showed hyphae with septa. Microbiologic culture showed A. fumigatus. We diagnosed the case as simple aspergilloma and began conventional amhotericin B treatment. Patient has allergic rash after amphotericin B infusion and on third day of hospitalisation he has abdominal pain. Despite his symtoms, hepatic transaminases were within normal range. Acute chloecycystitis was diagnosed after general surgey consultation and abdominal ultrasonography showed sludge in the gallbladder. We decided on caspofungin treatment of intravenous loading dose (70 $\mathrm{mg}$ /day) followed by $50 \mathrm{mg}$ /day infusion once daily. Patient was evaluated by chest surgery consultation and it was decided to wait for thoracic surgery consultation because of the abdominal complaints. Chloecystitis regressed in one week by medical treatment. Patient was operated at third week of antifungal treatment. Apical wedge resection was performed to remove the cavitary lesion and the fungus ball. Pathologic examination of surgical specimen showed hyphae with septa, brochial invasion and interstitial pulmonary disease (Figure 2). Antifungal treatment with caspofungin continued by intravenous route for five weeks after surgery. After eighth week of hospitalisation patient was discharged with itraconazole $200 \mathrm{mg}$ twice daily peroral route. At 12 weeks, antifungal treatment was stopped. Patient's symptoms such as cough, hemoptysis and productive sputum were not seen after tenth week of treatment.

\section{DISCUSSION}

Three classes of antifungal drugs are available for the treatment of aspergillosis: polyens, azoles and echinocandins. Echinocandins have activity against Aspergillus spp, but have a limited role because they have not been studied for initial treatment of invasive aspergillosis. Echinocandins are used for treatment of

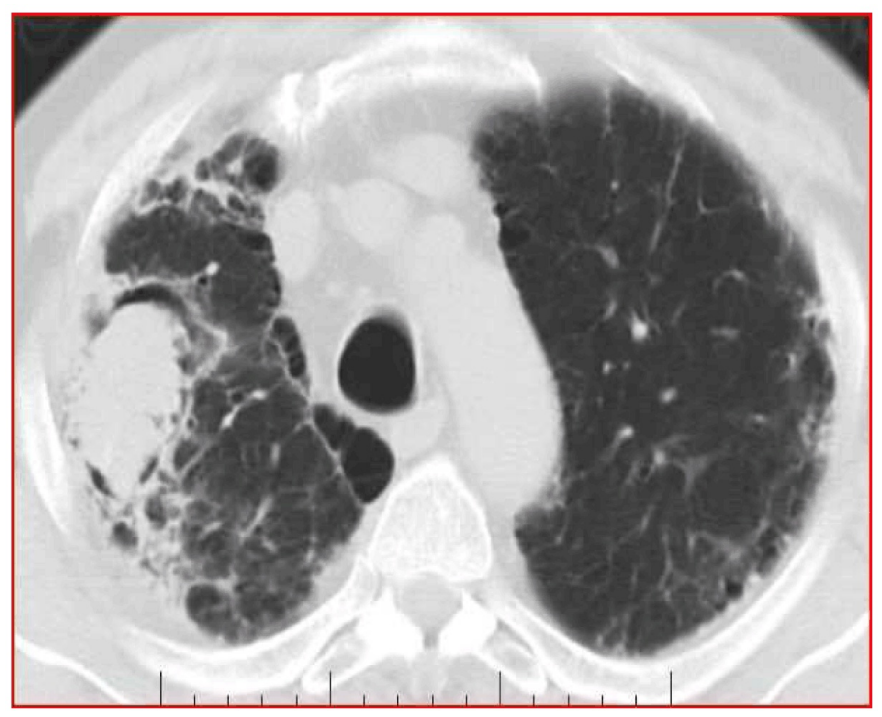

Figure 1: Air cresent formation with fungus ball in the lungs.

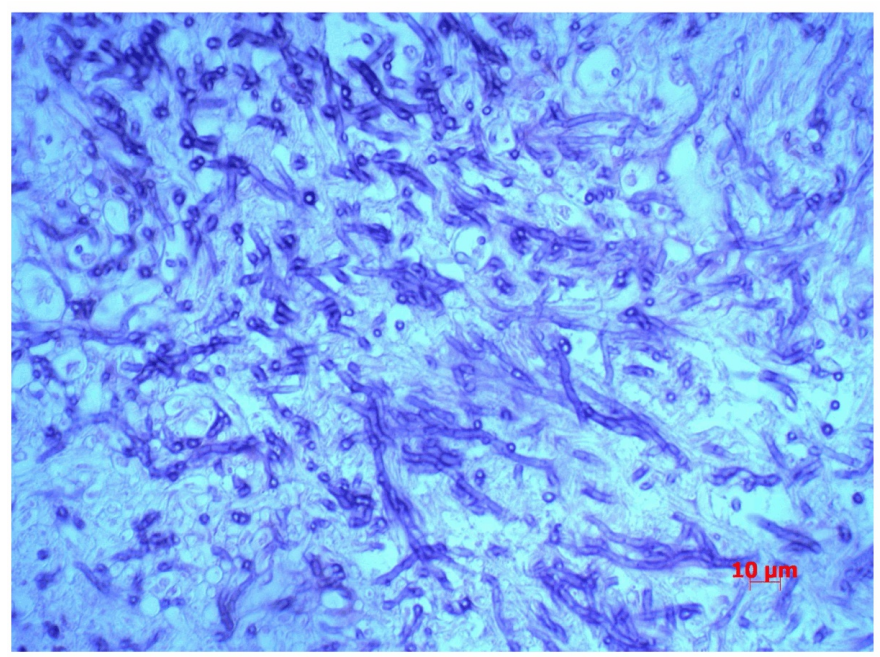

Figure 2: Histopathologic examination shows hyphae with septa, characteristics of aspergillosis (H\&E, x 200). 
invasive candidiasis especially in critically ill patients and less commonly for invasive aspergillosis especially as salvage treatment. The major advantages of echinocandins relative to other antifungal drugs are their fungicidal activity against Candida spp including fluzole resistant species combined with their relatively low potential for renal or hepatotoxicity [5]. Growth of Aspergillus species is inhibited even by very low concentrations in vitro with the effects predominantly observed at apical sub-apical branching where cell wall remodelling and beta glucan synthase are most active $[6,7]$. Azoles are the first agents of choice for pre- and post-operative antifungal therapy. In our patient allergic rash occured at the begining of treatment with conventional amphotericin B. As amphotericin B-lipid complex was not available and due to patient's cholecystitic symptoms such as nausea and vomiting antifungal treatment option was swicthed to caspofungin. Caspofungin was used in standard dose of $70 \mathrm{mg}$ on first day and subsequent dosing was 50 $\mathrm{mg}$ /day. In this case we prefered to use caspofungin as initial treatment especially because of the abdominal symptoms. After one week of treatment we made an interval rewieving of treatment for using caspofungin alone or in combination. We made a decision of using caspofungin alone because of improving clinical condition of the patient. Antifungal treatment after surgery of aspergillomas has been strongly recommended by most of the authors [8]. Antifungal treatment options after surgery depend on patient's clinical situation. Itraconazole and voriconazole are first line agents for oral antifungal treatment of aspergillosis [8]. Duration of antifungal therapy is not well defined in literature. Some authors have recommended lifelong therapy beacuse of the relapses [9]. Others suggest that a four week course of therapy post-operatively is reasonable, if the aspergilloma was fully resected without spillage. If there is spillage, then a minimum of 12 weeks therapy is recommended in an attempt to prevent pleural aspergillosis [8]. In our case, we used itraconazole for seven weeks after caspofungin intravenous therapy.

Risks and benefits of medical and surgical therapy vary with the manifestations of the disease and patients pulmonary status. Surgery is the mainstay of treatment for symptomatic patients with simple aspergilloma to prevent or treat life-threating hemoptysis [8]. Surgery of pleural based aspergilloma will be risky. Most of the surgens prefer lobectomy or cavernectomy beacuse of the pleural hemorrhagic complications. In our case we prefered pleural based wedge resection of cavitary lesion in order to preserve patient's vital capacity in the future.

\section{CONCLUSION}

Aspergilloma is a fungus ball composed of aspergillus hyphae, fibrin, mucus, and cellular debris found within a pulmonary cavity. In the treatment of aspergilloma, surgery and antifungal therapy must be given together. Choice of antifungal agents is the main cause of successful treatment. Caspofungin shows satisfactory antifungal activity against aspergilloma before and after surgery despite inadequate data about its use.

$* * * * * * * * *$

\section{Author Contributions}

Alper Sener - Substantial contributions to conception and design, Acquisition of data, analysis and interpretation of data, Drafting the article, revising it critically for important intellectual content, and Final approval of the version to be published

Senol Kobak - Substantial contributions to conception and design, Acquisition of data, Revising it critically for important intellectual content, Final approval of the version to be published

Timucin Alar - Substantial contributions to conception and design, Acquisition of data, Revising it critically for important intellectual content, Final approval of the version to be published

Ugur Gonlugur - Analysis and interpretation of data, Revising it critically for important intellectual content, Final approval of the version to be published

Asli Muratli - Analysis and interpretation of data, Revising it critically for important intellectual content, Final approval of the version to be published

\section{Guarantor}

The corresponding author is the guarantor of submission.

\section{Conflict of Interest}

Authors declare no conflict of interest.

\section{Copyright}

(C) Alper Sener et al. 2013; This article is distributed under the terms of Creative Commons Attribution 3.0 License which permits unrestricted use, distribution and reproduction in any means provided the original authors and original publisher are properly credited. (Please see www.ijcasereportsandimages.com/copyright-policy.php for more information.)

\section{REFERENCES}

1. Zmeili OS, Soubani AO. Pulmonary aspergilosis: a clinical update. QJM 2007 June;100(6):317-34.

2. Segal BH. Aspergillosis. N Engl J Med 2009 Apr 30;360(18):1870-84.

3. Douglas CM. Fungal beta(1,3)-D-glucan synthesis. Med Mycol 2001;39 Suppl 1:55-66.

4. Pappas PG, Kauffman CA, Andes D, et al. Clinical practice guidelines for the management of candidiasis: 2009 update by the Infectious Diseases Society of America. Clin Infect Dis 2009;48(5):503-35.

5. McCormack PL, Perry CM. Caspofungin: a review of its use in the treatment of fungal infections. Drugs 2005;65(14):2049-68. 
6. Beauvais A, Bruneau JM, Mol PC, Buitrago MJ, Legrand R, Latgé JP. Glucan synthase complex of Aspergillus fumigatus. $\mathrm{J}$ Bacteriol 2001;183(7):2273-9.

7. Bowman JC, Hicks PS, Kurtz MB, et al. The antifungal echinocandin caspofungin acetate kills growing cells of Aspergillus fumigatus in vitro. Antimicrob Agents Chemother 2002;46(9): 3001-12.

8. Walsh TJ, Anaissie EJ, Denning DW, et al. Treatment of aspergillosis: clinical practice guidelines of the Infectious Diseases Society of America. Clin Infect Dis 2008;46(3):327-60.

9. Denning DW, Riniotis K, Dobrashian R, Sambatakou H. Chronic cavitary and fibrosing pulmonary and pleural aspergillosis: case series, proposed nomenclature change, and review. Clin Infect Dis 2003;37 Suppl 3:S265-80.
Access full text article on other devices

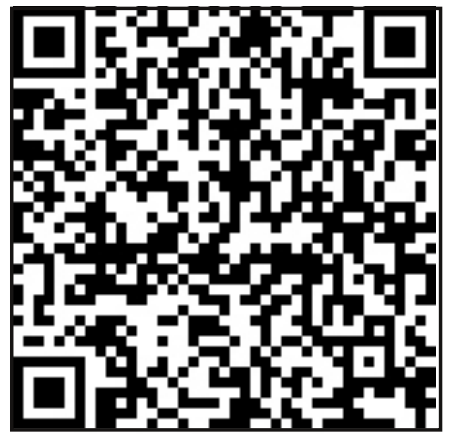

Access PDF of article on other devices

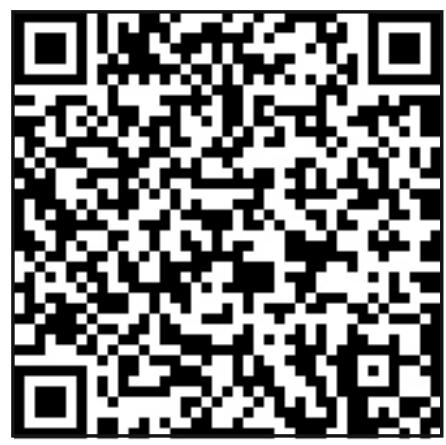

